\title{
Characterization of Programmers with Dyslexia
}

\author{
José L. Fuertes ${ }^{1}$, Luis F. González², and Loïc Martínez ${ }^{1}$ \\ ${ }^{1}$ Universidad Politécnica de Madrid, Spain \\ \{jfuertes, loic\} dfi.upm.es \\ ${ }^{2}$ Politécnico Colombiano Jaime Isaza Cadavid, Colombia \\ lfgonzaleza@elpoli.edu.co
}

\begin{abstract}
Computer programmers with dyslexia can be found in a range of academic and professional scenarios. Dyslexia may have the effect on a computer programmer of degrading the expected results during collaborative software development. These people may perform better using visual programming languages. However, we need to understand what programmers with dyslexia experience in order to be able to come up with possible solutions. We have con-ducted an analysis of existing literature and a survey on dyslexia and programming. This paper reports the preliminary results based on the data gathered so far and the key characteristics and needs of this group with the aim of defining the profile of computer programmers with dyslexia.
\end{abstract}

\section{Introduction}

Changes in inclusion due to policies such as the Convention on the Rights of Persons with Disabilities [1], make it possible for persons with dyslexia to become computer programmers. Dyslexia is a specific reading disorder, where "Reading comprehension skill, reading word recognition, oral reading skill, and performance of tasks requiring reading may all be affected. Specific developmental disorders of reading are commonly preceded by a history of disorders in speech or language development" [2].

A computer programmer with Dyslexia may have a degraded performance using traditional programming languages and may perform better using visual programming languages. However, we need to understand their profile as programmers in order to come up with possible solutions. So, an analysis of the literature and an electronic survey have been conducted.

\section{Related Work}

There is literature and software applications aimed at helping children with dyslexia. However, dyslexia among software developers has not been much researched, and we have not come across any software tool designed to help computer programmers with dyslexia in the process of creating computer programs. 
A literature review has identified two relevant studies. The first claims that dyslexic programmers usually come up against a number of difficulties when developing software [3]: remembering code details, sticking to a good code presentation layout, correctly defining variables correctly and being at coding and at debugging... How-ever this group of people have a great capacity for analysis, as usually they have an IQ above the average. The second study describes problems associated with dyslexia in the programming area in higher education [4]. They confirm problems described by [3] and they provide some accessibility guidelines for programmers with dyslexia.

Afterwards, we have identified the characteristics of adults with dyslexia [5]: they may have different learning-related symptoms, which show up as problems with reading, writing, organization, speech or mathematics. They may experience visuospatial difficulties (problems with mathematics, reading, writing and organization), speech sound difficulties (problems with speaking, reading, writing and organization) and correlating difficulties (problems with writing).

\section{$3 \quad$ Results of the Survey}

We have built a survey to determine the profile of computer programmers with dyslexia. The survey has been answered by Spanish-speaking computer programmers selected by a combination of convenience sampling and purposive snowball sampling. They were inquired sociodemographic, behavioral and programming variables [6].

The sample of 155 programmers had an average age of 22 years (from 20 to 44). $6.5 \%$ of all respondents believe that they are dyslexic $(\mathrm{n}=10)$. Of this group $40 \%$ consider that dyslexia has a negative effect on their programming performance. It is striking that only $20 \%$ of these 10 have been professionally diagnosed as being dyslexic, which probably means a lack of awareness and proper treatment of this disability.

Regarding behavioral variables (table 1 ), we found that $70 \%$ of computer programmers with dyslexia suffer anxiety when programming compared to $37.9 \%$ of programmers without dyslexia. Poor concentration does not appear to be a problem in the surveyed sample, as stated by $80 \%$ of computer programmers with dyslexia and $68.3 \%$ of programmers without dy slexia. However, these data contrast with the $60 \%$ of programmers with dyslexia and the $50.3 \%$ of programmers without dyslexia that responded that they were easily distracted when studying. On the other hand, $50 \%$ of the programmers with and $61.3 \%$ of the programmers without dyslexia stated that they do not often suffer mood swings. With respect to depression, $90 \%$ of the programmers with and $78.7 \%$ of programmers without dyslexia do not suffer from this. $60 \%$ of people with dyslexia in contrast to $23.4 \%$ of those without dyslexia consider themselves anxious persons. We also observed that $80 \%$ of programmers with and $68.3 \%$ of programmers without dyslexia do not suffer from insomnia. We found that $50 \%$ of programmers with and $55.2 \%$ programmers without dyslexia have trouble getting up in the morning.

In regard to programming activity, only $30 \%(\mathrm{n}=3)$ of programmers with and $20 \%$ $(n=29)$ of programmers without dyslexia have ever used a visual programming language. We found that $66.6 \%$ of programmers with dyslexia state that they make more mistakes programming using a textual language against $33.3 \%$ who state that they make 
more errors using a visual programming language. Table 2 shows the most representative data with respect to programmer preference for, ease of use of and speed using a visual language.

We have applied a chi-squared test to the variable "Do you think you are dyslexic?" with all the behavioral variables. We found that there is a statistically significant relationship for the variables: behavior to get attention $(\mathrm{p}=0.02)$, compulsive behavior $(p=0.016)$, excuses for not going to school $(p=0.03)$, programming hypoactivity disor$\operatorname{der}(p=0.026)$, frequent state of isolation in programming $(p=0.047)$, hyperactivity disorder $(p=0.019)$, anxious behavior $(p=0.01)$ and distorted vision $(p=0.0)$.

Table 1. Percentages of disagreement with respect to behavioural variables.

\begin{tabular}{|c|c|c|c|}
\hline $\begin{array}{l}\text { Percentage of disagreement with respect to behavioural } \\
\text { variables }(n=155)\end{array}$ & $\begin{array}{l}\text { PwithD } \\
(\mathrm{n}=10)\end{array}$ & $\begin{array}{l}\text { PwithoutD } \\
(\mathrm{n}=145)\end{array}$ & $\mathbf{p}$ \\
\hline I often made any number of excuses to get of going to school & $80.0 \%$ & $86.2 \%$ & 0.030 \\
\hline I now often make excuses to get out of going to work or university & $80.0 \%$ & $83.5 \%$ & 0.618 \\
\hline I often resort to attention-seeking behaviour & $70.0 \%$ & $85.5 \%$ & 0.002 \\
\hline fer episodes of irritability & $40.0 \%$ & $\%$ & 0.196 \\
\hline I consider myself to be an aggressive person & $80.0 \%$ & $83.5 \%$ & 0.065 \\
\hline self to be a withdrawn person & $90.0 \%$ & $67.6 \%$ & 0.420 \\
\hline elf compulsive person & $50.0 \%$ & $55.2 \%$ & 0.016 \\
\hline from others & $60.0 \%$ & $62.1 \%$ & 0.817 \\
\hline self hyperactive person & $40.0 \%$ & $64.8 \%$ & 0.019 \\
\hline I an & $50.0 \%$ & 71 & 0.270 \\
\hline My self-e & $60.0 \%$ & $60.7 \%$ & 0.899 \\
\hline I suffer from disordered vision & $70.0 \%$ & $95.9 \%$ & 0.000 \\
\hline I often suffer from fatigue & $40.0 \%$ & $66.2 \%$ & 0.143 \\
\hline
\end{tabular}

Table 2. Characteristics of affinity for visual programming

\begin{tabular}{lccc}
\hline Affinity for visual programming $(\mathbf{n}=32)$ & PwithD $(\mathbf{n}=\mathbf{3})$ & PwithoutD $(\mathbf{n}=\mathbf{2 9})$ & $\mathbf{p}$ \\
\hline I prefer a visual language & $66.7 \%$ & $24.1 \%$ & 0.746 \\
I find a visual language easier to use & $66.7 \%$ & $34.4 \%$ & 0.933 \\
I program faster with a visual language & $66.7 \%$ & $48.2 \%$ & 0.869 \\
\hline
\end{tabular}

\section{$4 \quad$ Analysis of the Results}

The percentage of the sample (6.5\%) stating that they believe that they are dyslexic is the range of adult population with dyslexia that goes from $5 \%$ to $10 \%$ [7].

It is usually considered that adults with dyslexia have obvious symptoms of low selfesteem and no confidence and feel helpless in some life situations [8], but the result for these variables, after applying a chi-squared test, confirm that these emotional variables do not play a role in defining their profile. The percentages related to performance, preference and ease of use of textual programming languages [3] confirm claims that programmers with dyslexia using programming tools with visual or graphic support are $20 \%$ better than programmers without dyslexia at developing computer programs. We 
did find that programmers with dyslexia make fewer mistakes than programmers without dyslexia when using a visual programming language.

\section{$5 \quad$ Conclusions and Future Work}

The emotional characteristics proposed in [8] to define the profile of adults with dyslexia seem not to be relevant to define the profile of programmers with dyslexia. However, further research is required to explore other variable types.

We have also found that programmers with dyslexia have a preference for visual programming, as also explained in [3], and they claim to make fewer mistakes.

We intend to gather more replies to our survey, by sending it to programmers in other Spanish-speaking countries and to programmers that belong to associations of persons with dyslexia. Then we plan to broaden the spectrum and perform the same study with English-speaking programmers to get a broader understanding of issues.

In the long term, and with the aim of counteracting the performance differences in interactive programming, we propose to design a two-way visual and textual code transformation model. The goal is to develop software on a single interface that inter-acts between the two codes, providing support for programmers with dyslexia in the process of interacting with their teammates.

\section{References}

1. UN (2006). Convention on the rights of persons with Disabilities. United Nations.

2. WHO (2011). ICD-10 international statistical classification of diseases and related health problems. Volume 2. Introduction manual. Malta: World Health Organisation publications.

3. Dixon, M. (2007): Comparative study of disabled vs. non-disabled evaluators in user-testing: Dyslexia and first year students learning computer programming. In: C. Stephanidis (Ed.). Springer Berlin Heidelberg, 647-656

4. Stienen-Duran, S., \& George, J. (2014). Supporting dyslexia in the programming classroom. 5th International Conference on Software Development and Technologies for Enhancing Accessibility and Fighting Info-Exclusion, Procedia Computer Science, 419-430.

5. Ingram, T. (1964). "The dyslexic child", The Practitioner, 192, 503-516.

6. González, L. (2016). Encuesta tesis doctoral "transformación bidireccional de código de software visual y texto, para mitigar los efectos de la dislexia en el desarrollo de software" (in Spanish). Available at http:// oa .upm.es/39155/

7. Everatt, J., Smythe, I., Ocampo, D., \& Gyarmathy, E. (2004). Issues in the assessment of literacy-related difficulties across language backgrounds: A cross-linguistic comparison. Journal of Research in Reading, 27, 141-151.

8. Shaywitz, S. E. (1996). Dyslexia. Scientific American, 275(5), 98-104 